\title{
Bimolecular recombination in a low bandgap polymer:PCBM blend solar cell with a high dielectric constant
}

Guanran Zhang, Tracey M. Clarke* and Attila J. Mozer*

G. Zhang, A/Prof. A. J. Mozer

Intelligent Polymer Research Institute and the ARC Centre of Excellence for Electromaterials

Science, University of Wollongong, North Wollongong, NSW 2500, Australia

Dr. T. M. Clarke

Department of Chemistry, University College London, WC1H 0AJ, United Kingdom

E-mail: tracey.clarke@ucl.ac.uk, attila@uow.edu.au

Keywords: dielectric screening, polymers, organic solar cell, recombination

\begin{abstract}
The strength of dielectric screening is one of the most intriguing yet least studied contributing factors to the operation and performance limit of organic solar cell devices. Increasing the dielectric constant of semiconducting polymers may close the performance gap between inorganic and organic solar cell devices. Here, a dielectric constant of 16.7 is reported for a DPP-based low bandgap polymer DT-PDPP2T-TT and 7 for its 1:3 blend with [60]PCBM ([6,6]-phenyl-C61-butyric acid methyl ester) using frequency and voltage dependent capacitance and charge extraction by linearly increasing voltage techniques. The charge mobility within the blend device $\left(1.8 \times 10^{-3} \mathrm{~cm}^{2} \mathrm{~V}^{-1} \mathrm{~s}^{-1}\right)$ is found to be among the highest reported. Bimolecular recombination and charge carrier lifetime in efficient photovoltaic devices at large
\end{abstract}


active layer thicknesses are measured and compared to poly (3-hexylthiophene) (P3HT):PCBM $(1: 1 \mathrm{w} / \mathrm{w})$ and poly[2,6-(4,4-bis-(2-ethylhexyl)-4H-cyclopenta[2,1-b;3,4-b']dithiophene)-alt4,7(2,1,3-benzothiadiazole)] (PCPDTBT):PCBM (1:2 w/w) devices. When normalized to mobility, the bimolecular recombination coefficient in DT-PDPP2T-TT:PCBM is a factor two lower than in P3HT:PCBM and an order of magnitude lower than in PCPDTBT:PCBM. The recombination mechanism is found to be close to diffusion-controlled Langevin recombination. The slower recombination and longest charge carrier lifetime are explained from a smaller coulomb capture radius, which, together with increased diffusion coefficient, leads to efficient charge extraction in photovoltaic devices with active layer thicknesses approaching $300 \mathrm{~nm}$.

\section{Introduction}

Conjugated polymers as photoactive components in bulk heterojunction polymer solar cells have a dielectric constant $\left(\varepsilon_{r}\right)$ in the range of 3-5.7. ${ }^{[1]}$ The fullerene derivative [6,6]-phenylC61-butyric acid methyl ester ([60]PCBM) has a dielectric constant of 3.9. ${ }^{[2]}$ The highest reported dielectric constant in polymer: PCBM blends reported so far is $4.95 .{ }^{[3]}$ The generally low dielectric constant is indicative of weak dielectric screening, which has several consequences related to the operation and performance of polymer solar cells. Firstly, the exciton binding energy between the photo-excited electron and hole is expected to be large. For example, using a modified Onsager theory where the electron-hole pair dissociation takes place when random energetic disorder (here taking an estimate of $100 \mathrm{meV}$ ) equals the Coulomb energy, the Coulomb capture radius is $4.8 \mathrm{~nm}$ at $\varepsilon_{r}$ of 3 and when $\varepsilon_{r}$ increases to 10 the Coulomb capture radius decreases to $1.4 \mathrm{~nm}$ (Table S1). The dissociation probability $P(E)$ is predicted to increase significantly over 2 magnitudes when dielectric constant increases, suggesting more efficient charge separation. Also, efficient charge separation requires that 
the energy offset between the lowest oxidation potential of the conjugated polymer and the first reduction potential of the electron acceptor matches or exceeds the exciton binding energy, which can lead to significant energetic loss (up to $10 \%$ in case of $0.4 \mathrm{eV},{ }^{[1 \mathrm{~d}]}$ ) in the maximum obtainable power conversion efficiency. Such energetic loss is likely reduced with higher dielectric constant. Secondly, large Coulomb attraction force between the electron and hole pairs (geminate or non-geminate) leads to strong recombination requiring a larger driving force for charge extraction. Carrier loss due to recombination leads to reduced fill factor and open circuit voltage $V_{O C}$, especially when large active layer thicknesses are used. ${ }^{[3]}$ Thirdly, weak dielectric screening leads implies small polaron transport mechanism with strong electric field and temperature dependence of mobility. For example, mobility values tend to be low, on the order of $10^{-5}-10^{-4} \mathrm{~cm}^{2} \mathrm{~V}^{-1} \mathrm{~s}^{-1},{ }^{[4]}$ further contributing to active layer thickness limitations (optimal thickness values around 80-100 $\mathrm{nm}$ for most polymer solar cells with a few exceptions, ${ }^{[5]}$ ). Fourthly, Koster et al. suggested that weak dielectric screening leads to a large singlet-triplet energy gap, which leads to additional recombination pathways to the lowest energy triplet state from the charge separated state. ${ }^{[1 \mathrm{~d}]}$

Enhancing dielectric screening by molecular design has been proposed to solve some of the most important limitations of polymer solar cells. Koster et al. suggested that organic materials with a dielectric constant above 9 could potentially close the performance gap between organic and inorganic semiconductors. ${ }^{[1 \mathrm{~d}]}$ However, there is no general design guideline successfully demonstrated that lead to significantly increased dielectric screening in semiconductive polymers. Side chain design with fluorine substitution and other polar segments were suggested as possible routes leading to moderate increases.

Cho et al. synthesized a diketopyrrolopyrrole (DPP) based polymer PIDT-DPP-CN with dielectric constant of $5(1000 \mathrm{~Hz})$ by introducing a polar nitrile side-chain. ${ }^{[6]}$ Comparing to the 
counterpart with none-polar side-chain, increase in Voc, fill factor and efficiency was observed. A longer small-perturbation lifetime, attributed to reduced bimolecular recombination, was reported. Similar approach of side chain modification was used by Torabi et al. to increase dielectric constant without sacrificing mobility or solubility. ${ }^{[2]}$ Lu et al. increased the dielectric constant of triophene-quinoxaline (TQ) from 4.5 to $5.7(1000 \mathrm{~Hz})$ by fluorination. ${ }^{[1 \mathrm{e}]}$ The fluorinated copolymer FTQ showed a larger band gap and thus an increase in $V_{O C \text {. The highest }}$ dielectric constant of 15 reported for organic materials is from a macromolecule of hyperbranched copper phthalocyanine $(\mathrm{HBCuPc})$ film, the high dielectric constant of which is suggested to benefit from long-range delocalization of polarons. ${ }^{[7]}$

Reports of the effect of dielectric screening on charge separation, voltage loss and recombination are also scarce. Chen et al. attributed the $V_{O C}$ loss across seven different polymer:PCBM systems to two main sources: i) bimolecular recombination and ii) free energy loss of the photo-excited state due to strong exciton binding. ${ }^{[3]}$ They reported that with dielectric constant between 3.36 and 4.95, the bimolecular recombination loss is similar for all systems (in the range of 0.1-0.2 eV), while the free energy loss due to weak dielectric screening is reduced significantly from $0.34 \mathrm{eV}\left(\varepsilon_{r}=3.36\right)$ to below $0.01 \mathrm{eV}\left(\varepsilon_{r}=4.95\right)$.

As clear from the above literature review, there are very few studies comparing dielectric constant with bimolecular recombination strength in polymer solar cells, limited by the availability of polymers with strong dielectric screening. In this work, we report a dielectric constant 16.7 for a DPP-based low bandgap polymer DT-PDPP2T-TT (1-Materials, see Figure S1 for structure). ${ }^{[8]}$ When mixed with $[60] \mathrm{PCBM}$ in 1:3 w/w ratio, a dielectric constant of seven is measured in the photovoltaic blend. The charge carrier mobility in the blend measured by photo-CELIV was found to be one of the highest reported in the literature $\left(1.8 \times 10^{-3} \mathrm{~cm}^{2} \mathrm{~V}^{-1} \mathrm{~s}^{-}\right.$ $\left.{ }^{1}\right)$. These properties make the DT-PDPP2T-TT system an interesting candidate to study the 
correlation between dielectric constant, mobility and bimolecular recombination. For this purpose, devices with over $4 \%$ efficiency were fabricated with high fill factor of 0.62 achieved at large active layer thickness of $267 \mathrm{~nm}$. Bimolecular recombination coefficient and charge carrier lifetime are compared with well-known, low dielectric constant polymer: PCBM blends of P3HT:PCBM (1:1,w/w) and PCPDTBT:PCBM (1:2, w/w). These systems are selected for comparison because of the well-established recombination kinetics ${ }^{[9]}$ as well as their low dielectric constant values, which provides a good contrast to the high dielectric constant of DTPDPP2T-TT:PCBM. The comparison reveals the benefit of stronger dielectric screening in the lowest bimolecular recombination coefficient normalized to charge mobility in DT-PDPP2TTT:PCBM, which is explained by smaller Coulomb capture radius. The dielectric constant of the polymer:fullerene blend found to be the average value of the polymer and PCBM. The increased dielectric constant of the polymer is currently unknown.

\section{Experimental Techniques}

Bias and frequency dependent capacitance: The parallel capacitance $C_{P}$ was obtained using impedance measurements over a voltage bias scan at a fixed frequency, assuming an equivalent circuit of a capacitor in parallel with a resistor. The parallel capacitance in this simple model has been shown to be an accurate estimation of the geometric capacitance at low frequencies below $10 \mathrm{kHz},{ }^{[10]}$ which is our range of interest. At higher frequencies, the series resistance (i.e. contact resistance, resistance of the external circuit) may affect the frequency dependency, producing a lower $C_{P}$ than the real value. The geometric capacitance is determined from the $C^{-}$ ${ }^{2}$-Voltage plot by extrapolating the linear region at reverse bias to the y axis $(V=0)$ and the intercept corresponds to $C_{P}^{-2}$. 
Charge Extraction by Linearly Increasing Voltage: A linear voltage ramp was applied to the devices at set frequency with a voltage ramp maximum altitude $U$ and a pulse duration $t_{\text {pulse }}$, while the device being covered in the dark to eliminate charge photogeneration. The current step response would reach $j(0)=C_{P} U / A t_{\text {pulse }}$ in the circuit time constant $\tau_{R C}$ for an $R C$ circuit, where $A$ is the size of the active area and $d$ is the active layer thickness. The time constant $\tau_{R C}$ was controlled below 200 ns by applying different resistors depending on the device capacitance.

Photogenerated Charge Extraction by Linearly Increasing Voltage: The devices were photoexcited at open circuit using a nanosecond switch. After a certain delay time, the switch was opened and a ramp voltage pulse was applied to the device with variable bias altitude $U$ and pulse duration $t_{\text {pulse }}$. During the delay time an adjustable offset was applied to compensate for the charge leakage prior to extraction. The extraction current will reach a maximum $\Delta j$ after time $t_{\max }$, from which the charge carrier mobility $\mu$ could be calculated for samples with moderate conductivity $(\Delta j \leq j(0)):^{[11]}$

$\mu=\frac{2 d^{2}}{3 A t_{\max }^{2}\left[1+0.36 \frac{\Delta j}{j(0)}\right]}$

Time-Resolved Charge Extraction: For TRCE measurements the devices were photo-excited using a laser pulse at open circuit using a nanosecond switch. ${ }^{[12]}$ After a certain delay time the switch was opened and the extraction transients at short circuit condition were recorded. After subtracting the dark response, the extracted charge density at a certain delay time was obtained from integration of the extraction transient. Time dependent bimolecular recombination coefficient $\beta$ was calculated from charge carrier density and the first order derivative of carrier density versus time using the equation for bimolecular (second order) recombination:

$\frac{d n}{d t}=-\beta n^{2}$ 
The charge carrier lifetime could also be calculated from $\beta$ :

$\tau=\frac{1}{n \beta}=-n\left(\frac{d n}{d t}\right)^{-1}$

The bimolecular recombination coefficient $\beta$ is often compared to the Langevin recombination coefficient $\beta_{L}$ :

$\beta_{L}=\frac{e \mu}{\varepsilon_{r} \varepsilon_{0}}$

Bulk-generation Time-of-Flight: TOF is used to obtain the ratio between $\beta$ and $\beta_{L}{ }^{[13]}$ This was achieved by measuring extraction transients at different excitation densities under a constant extraction bias. The integration of each extraction transient gave the extracted charge $Q_{e}$ under the specific excitation density. The extraction time $t_{e}$, which is the time required to deplete the reservoir under the applied bias, was determined by the difference of half-maximum decay time $t_{1 / 2}$ at high and low excitation densities. The bimolecular recombination reduction factor $\zeta$ was then calculated as

$\xi=\frac{\beta}{\beta_{L}}=\frac{t_{t r}}{t_{e}} \cdot \frac{C U_{0}}{Q_{e}}$

where $t_{t r}=\frac{d^{2}}{\mu \cdot U_{0}}$ is the transit time required for the carriers to travel through the sample thickness under the applied bias, and $d$ is the active layer thickness. By selecting the resistance $R$, the circuit $R C$ time constant was tuned so that the extraction transients were $R C$ limited (QTOF).

\section{Results}

\subsection{Dielectric constant}


Figure 1 shows the $C_{p}^{-2}$ plotted as a function of voltage for pristine and [60]PCBM blend devices using DT-PDPP2T-TT (chemical structure and device architecture illustrated in Figure $\mathrm{S} 1)$ measured under a range of applied bias from $-2 \mathrm{~V}$ to $2 \mathrm{~V}$ at $1 \mathrm{kHz}$. The inset shows a charge extraction by linearly increasing voltage (CELIV) transient measured at voltage pulse duration $t_{\text {pulse }}$ of $10 \mu$ s and maximum voltage $U$ of $2 \mathrm{~V}$. From the frequency modulated bias dependent capacitance measurements the capacitance of the pristine and blend films are obtained (3.08 $\mathrm{nF}$ and $1.38 \mathrm{nF}$, respectively). CELIV transient gives a geometric capacitance of $1.38 \mathrm{nF}$, identical to that obtained from the bias dependent capacitance plots. The dielectric constant is calculated using the obtained capacitance values assuming that the capacitance at $1 \mathrm{kHz}$ arises from the geometric capacitance of the device

$C=\varepsilon_{0} \varepsilon_{r} \frac{A}{d}$

An average thickness of $289 \pm 7 \mathrm{~nm}$ for the pristine film and $267 \pm 8 \mathrm{~nm}$ for the blend film was obtained. The active area of the devices was $0.06 \mathrm{~cm}^{2}$ defined by the overlap of contacts between the edged indium thin oxide (ITO) and evaporated aluminium top contact. The dielectric constant for the above DT-PDPP2T-TT:[60]PCBM 1:3 w/w blend device gives a $\varepsilon_{r}$ of $6.79 \pm 0.14$ and for the pristine polymer $\varepsilon_{r}=16.7 \pm 0.4$ is obtained. 


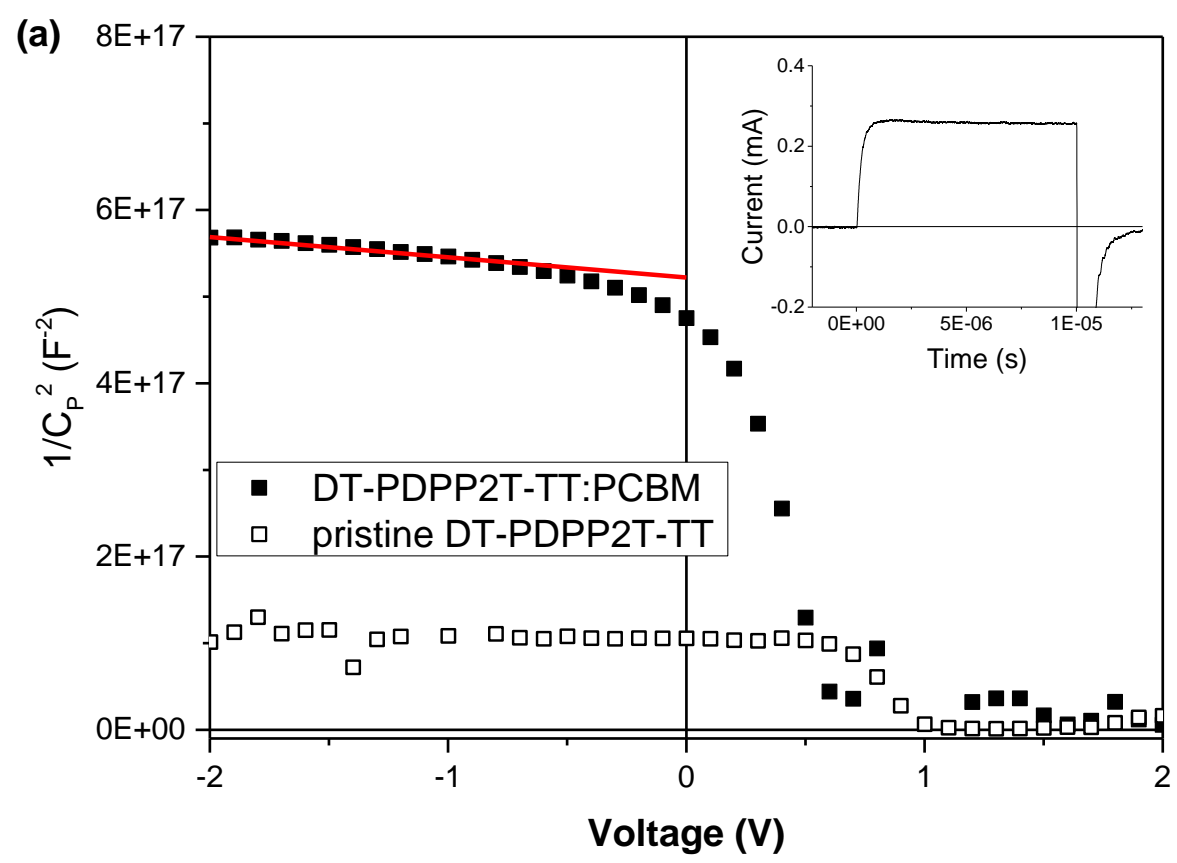

(b)

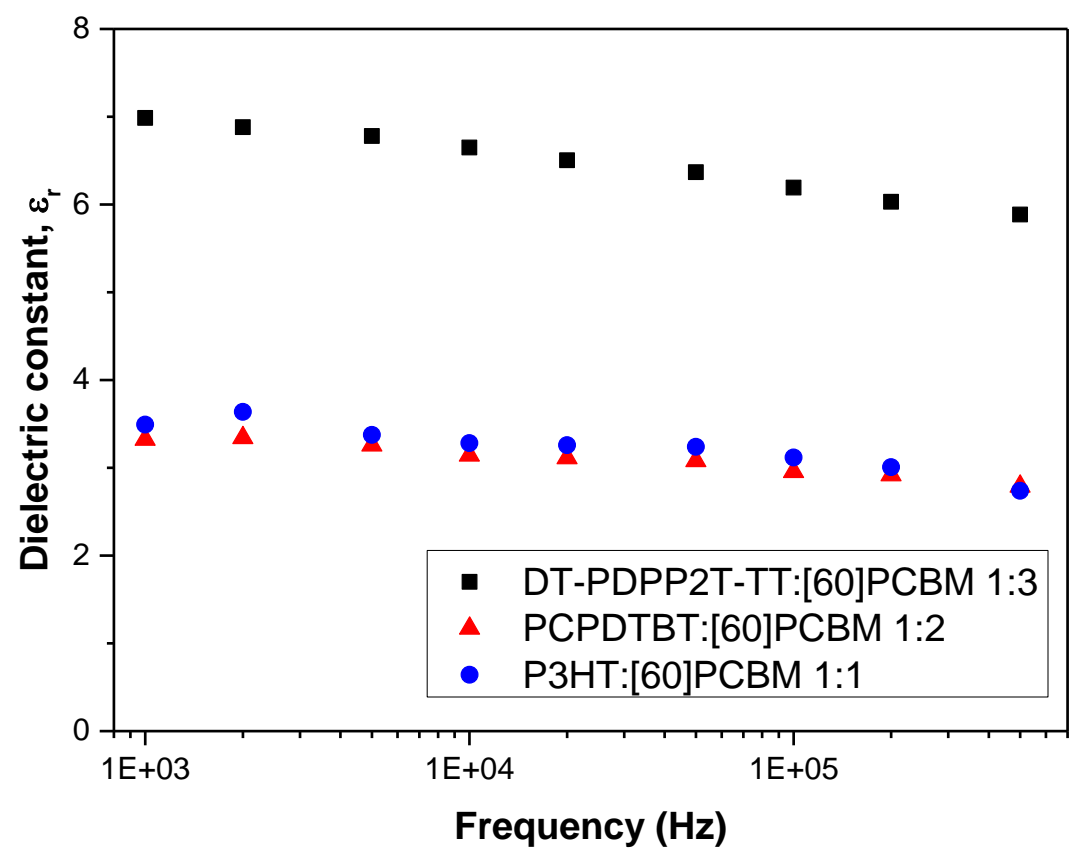

Figure 1. Voltage and frequency dependent capacitance measured using frequency modulated current-voltage measurements. (a) The $1 / C_{P}{ }^{2}$ plotted as a function of voltage bias for a $267 \mathrm{~nm}$ DT-PDPP2T-TT: PCBM 1:3 blend device as well as a $289 \mathrm{~nm}$ pristine film, measured using an $50 \mathrm{mV}$ voltage modulation at $1000 \mathrm{~Hz}$, and (b) dielectric constant calculated from $C_{P}$ using Equation 6 for DT-PDPP2T-TT: PCBM 1:3 w/w blend, PCPDTBT: PCBM 1:2 w/w blend as well as P3HT: PCBM 1:1 w/w blend, plotted as a function of frequency. The inset in (a) shows a dark CELIV curve of DT-PDPP2T-TT: PCBM measured at voltage pulse duration of $10 \mu \mathrm{s}$ and maximum voltage of $2 \mathrm{~V}$. 
The effect of active layer thickness, different polymer batches and possible contribution from neighbouring pixels has also been considered. Five devices of DT-PDPP2T-TT:PCBM (1:3, w/w) with different thickness were fabricated and the dielectric constant is calculated for each device using bias dependent impedance and CELIV techniques. Two single-pixel devices were fabricated, where only one aluminium strip was evaporated leading to only one pixel (See Figure S1b). These results are summarized in Table S2. An average dielectric constant of 7.29 \pm 0.75 for DT-PDPP2T-TT:[60]PCBM $(1: 3, \mathrm{w} / \mathrm{w})$ blend was calculated. DT-PDPP2TTT:[70]PCBM (1:3, w/w) devices were also fabricated, giving a dielectric constant of $6.85 \pm 0.88$.

Figure $1 \mathrm{~b}$ shows the frequency dependence of the dielectric constant calculated from the capacitance as above in DT-PDPP2T-TT:PCBM (1:3, w/w) blend, compared with that of 1:2 w/w PCPDTBT:PCBM blend and 1:1 w/w P3HT:PCBM blend. A weak frequency dependence of $\varepsilon_{r}$ is observed, where $\varepsilon_{r}$ decreased from 7.0 at $1 \mathrm{kHz}$ to 5.9 at $500 \mathrm{kHz}$. Similar frequency dependence is observed in PCPDTBT:PCBM as well as P3HT:PCBM blends.

\subsection{Charge carrier mobility}

Figure 2 shows photo-CELIV transients measured at various delay times (a) at $10 \mu \mathrm{J} \mathrm{cm}$ laser $^{-2}$ intensity and at various laser intensities (b) at a fixed $5 \mu$ s delay time for a photovoltaic device based on the DT-PDPP2T-TT: [60]PCBM (1:3, w/w) device. No strong dependence of the time to reach maximum extraction current $\left(t_{\max }\right)$ on delay time or excitation density was observed. Carrier mobility was calculated from 22 recorded transients by varying, in addition to light intensity and delay time, the voltage pulse duration $t_{\text {pulse }}(10 \mu \mathrm{s}-100 \mu \mathrm{s})$ and maximum applied voltage $U(0.1 \mathrm{~V}-5 \mathrm{~V})$. The average mobility value of $(1.8 \pm 0.6) \times 10^{-3} \mathrm{~cm}^{2} \mathrm{~V}^{-1} \mathrm{~s}^{-1}$ was obtained. 
The carrier mobility and dielectric constant in P3HT:PCBM and PCPDTBT:PCBM systems are summarized in Table 1, obtained using the same method as DT-PDPP2T-TT:PCBM.
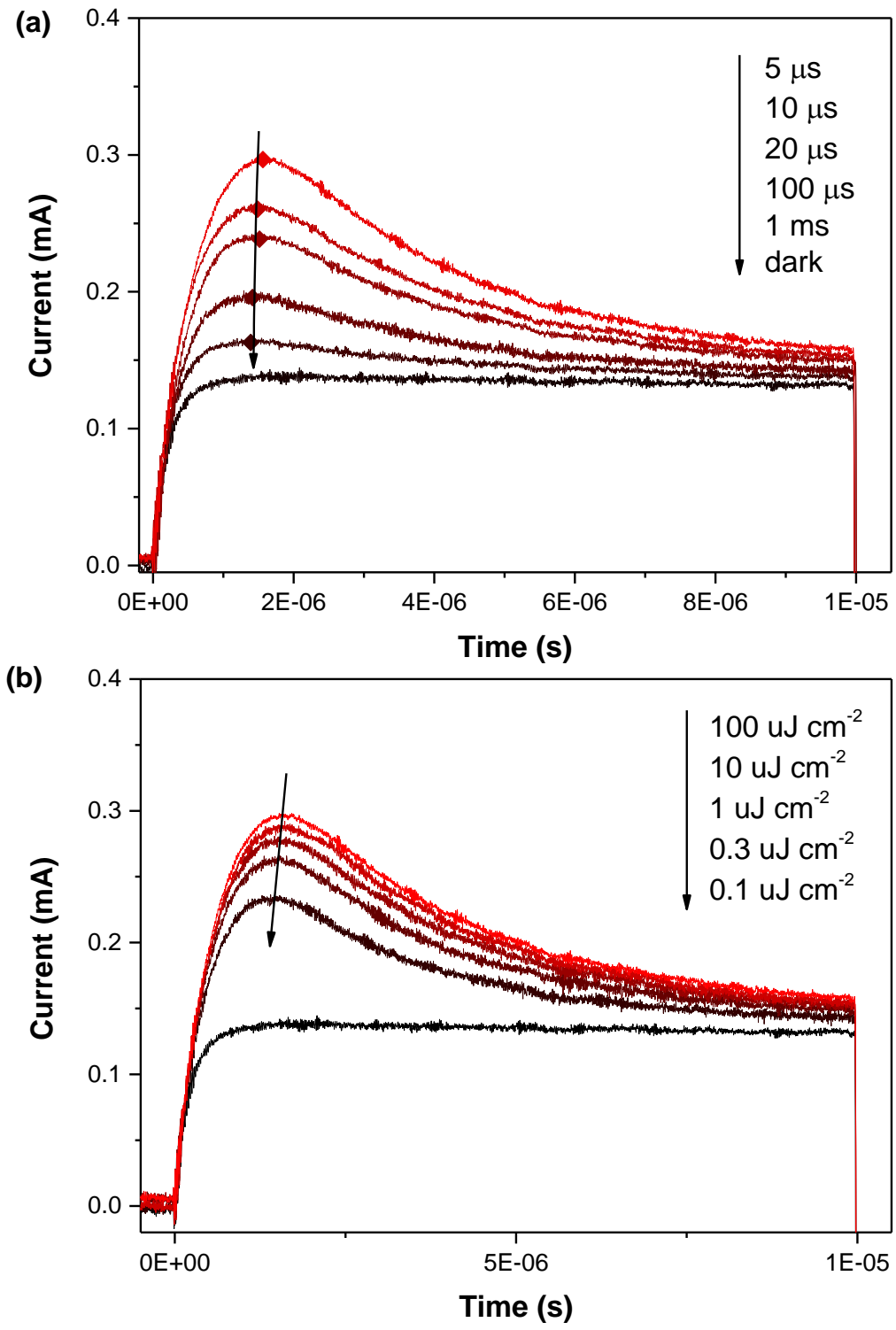

Figure 2. Photo-CELIV transients measured at (a) different delay times and (b) different excitation densities. Voltage pulse duration of $10 \mu$ s and maximum voltage of $2 \mathrm{~V}$ is used for all transients.

Table 1. Charge carrier mobility, dielectric constant and Langevin recombination coefficient for DT-PDPP2T-TT:PCBM, PCPDTBT:PCBM and P3HT:PCBM devices.

\begin{tabular}{lllll}
\hline Donor polymer & $\begin{array}{c}\text { Donor: acceptor } \\
\text { weight ratio }\end{array}$ & $\begin{array}{l}\text { Mobility } \\
\left(\mathrm{cm}^{2} \mathrm{~V}^{-1} \mathrm{~s}^{-1}\right)\end{array}$ & $\begin{array}{l}\text { Dielectric } \\
\text { constant, } \varepsilon_{r}\end{array}$ & $\begin{array}{l}\beta_{L} \\
\left(\mathrm{~cm}^{3} \mathrm{~s}^{-1}\right)\end{array}$ \\
\hline
\end{tabular}




\begin{tabular}{lllll}
\hline DT-PDPP2T-TT & $1: 3$ & $1.8 \times 10^{-3}$ & 7.3 & $4.7 \times 10^{-10}$ \\
PCPDTBT & $1: 2$ & $1.32 \times 10^{-4}$ & 3.3 & $6.7 \times 10^{-11}$ \\
P3HT & $1: 1$ & $4.49 \times 10^{-5}$ & 3.5 & $2.3 \times 10^{-11}$ \\
\hline
\end{tabular}

\subsection{Bulk-generation time-of-flight}

Figure S1c shows the current voltage curves of an ITO/PEDOT:PSS/DT-PDPP2TTT:PCBM/Al device, with active layer thickness of $267 \mathrm{~nm}$. The internal as well as external quantum efficiency is plotted in Figure S1d. The measured photovoltaic parameters are displayed in Table S3. The power conversion efficiency of this device is $4 \%$ with short circuit current of $9.2 \mathrm{mAcm}^{-2}$, open circuit voltage of $0.71 \mathrm{~V}$ and fill factor of 0.62.

Bulk-generation time-of-flight (TOF) was carried out on the device using external resistors of $1 \mathrm{k} \Omega$ and $10 \mathrm{k} \Omega$. The recorded transients, the ratio between extracted charge and capacitive charge $Q_{e} /{ }_{C U_{0}}$ and the time for the transient to decay to half its maximum magnitude $t_{1 / 2}$ plotted as a function of excitation density are shown in Figure S2. Transients measured using $1 \mathrm{k} \Omega$ and $10 \mathrm{k} \Omega$ both saturated with excitation density and neither show distinct feature of extraction time $t_{e}$. The $Q_{e} / C U_{0}$ ratio reached 2.7 at the highest excitation density using a $1 \mathrm{k} \Omega$ resistor, which is slightly higher than that using the $10 \mathrm{k} \Omega$ resistor. The $t_{1 / 2}$ values for the 10 $\mathrm{k} \Omega$ transients are approximately an order of magnitude higher than that of the $1 \mathrm{k} \Omega$ ones, while both showing weak excitation density dependence. The reduction factor $\beta / \beta_{L}$ is calculated using Equation 5, giving values of 0.07 using a $1 \mathrm{k} \Omega$ resistor and 0.015 using a $10 \mathrm{k} \Omega$ resistor.

\subsection{Charge density decay}

Time-resolved charge extraction (TRCE) measurements using a nanosecond switch were conducted on the above photovoltaic device. Charge density obtained from TRCE under 
different excitation densities plotted as a function of delay time is shown in Figure 3. A biphasic recombination behavior is observed, which consists of a strongly excitation density dependent phase from $500 \mathrm{~ns}$ to $10 \mu \mathrm{s}$ and an excitation density insensitive slow phase from $10 \mu$ s to $1 \mathrm{~ms}$. The slow phase was fitted to power law $\left(n \propto t^{-\alpha}\right)$ giving a power law gradient $\alpha$ of 0.16 . Close to identical power law kinetics is observed for all excitation densities above $0.1 \mu \mathrm{J} \mathrm{cm}^{-2}$ for the slow phase. Increasingly faster recombination kinetics with increasing excitation density is observed for the fast phase. The charge density increases with increasing excitation density up to $100 \mu \mathrm{J} \mathrm{cm}^{-2}$, where the charge density starts to saturate. The charge density values could be further correlated with Voc at each delay time by measuring corresponding photovoltage decay, see Figure S4.

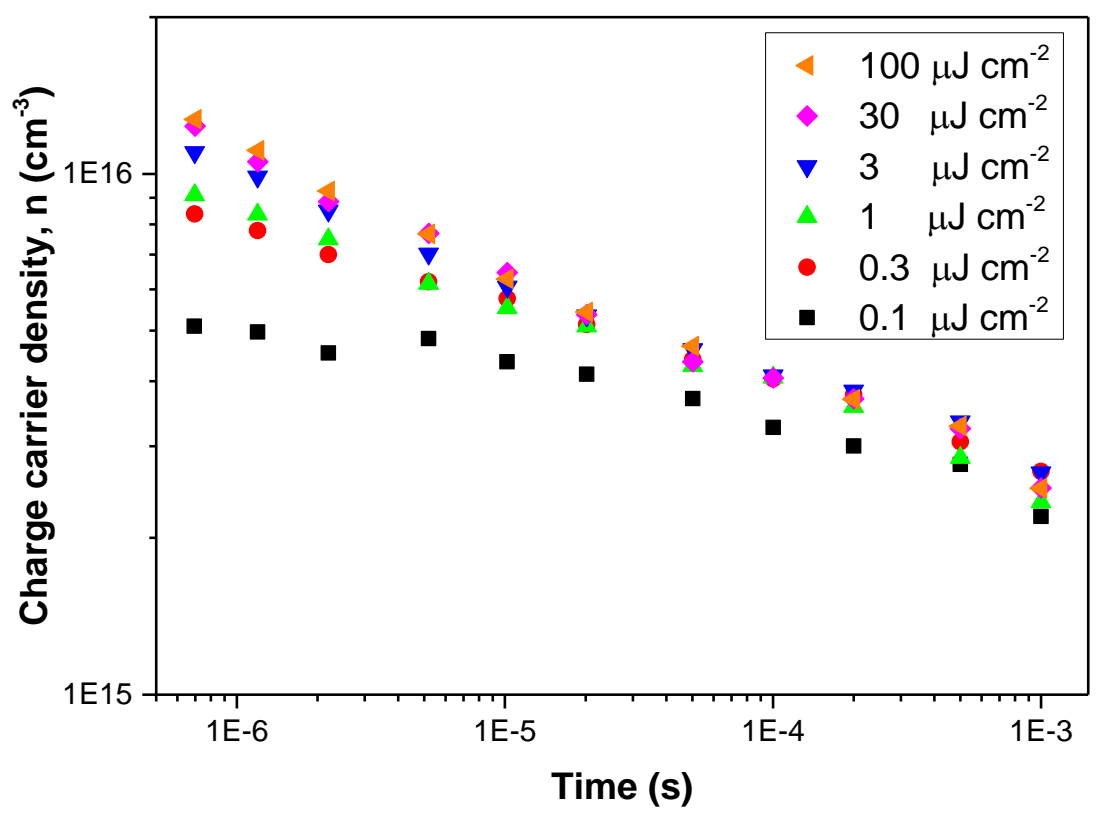

Figure 3. Charge density decay of DT-PDPP2T-TT:PCBM device measured at different delay times and under different excitation densities obtained from TRCE.

\subsection{Time-dependent bimolecular recombination coefficient}


Figure 4a shows the obtained $\beta$ plotted against delay time. The error bars show the variation with excitation density at the same delay time. The horizontal dash lines show the $\beta$ values obtained from bulk-generation time-of-flight (TOF) measurements under resistance of $1 \mathrm{k} \Omega$ and $10 \mathrm{k} \Omega$. The bimolecular recombination coefficient of DT-PDPP2T-TT:PCBM is compared with that of P3HT:PCBM (1:1, w/w) and PCPDTBT:PCBM (1:2, w/w) blend systems in Figure 4a. P3HT:PCBM system shows a $\beta$ of $1.07 \times 10^{-12} \mathrm{~cm}^{3} \mathrm{~s}^{-1}$ at $1 \mu \mathrm{s}$, which is over an order of magnitude lower than that of DT-PDPP2T-TT:PCBM. PCPDTBT:PCBM and DT-PDPP2TTT:PCBM show very similar $\beta$ values of $2.02 \times 10^{-11} \mathrm{~cm}^{3} \mathrm{~s}^{-1}$ and $1.95 \times 10^{-11} \mathrm{~cm}^{3} \mathrm{~s}^{-1}$ at $1 \mu \mathrm{s}$, respectively. A distinct difference in the time dependence of $\beta$ is observed between these three systems. The strongest time dependence is observed for the PCPDTBT:PCBM blend. At $10 \mu \mathrm{s}$, the $\beta$ of DT-PDPP2T-TT:PCBM is more than twice as large compared to that of PCPDTBT:PCBM. Much weaker time dependence is observed for P3HT:PCBM with $\beta$ decreasing only an order of magnitude over the $1 \mu$ s to $1 \mathrm{~ms}$ timescale. 

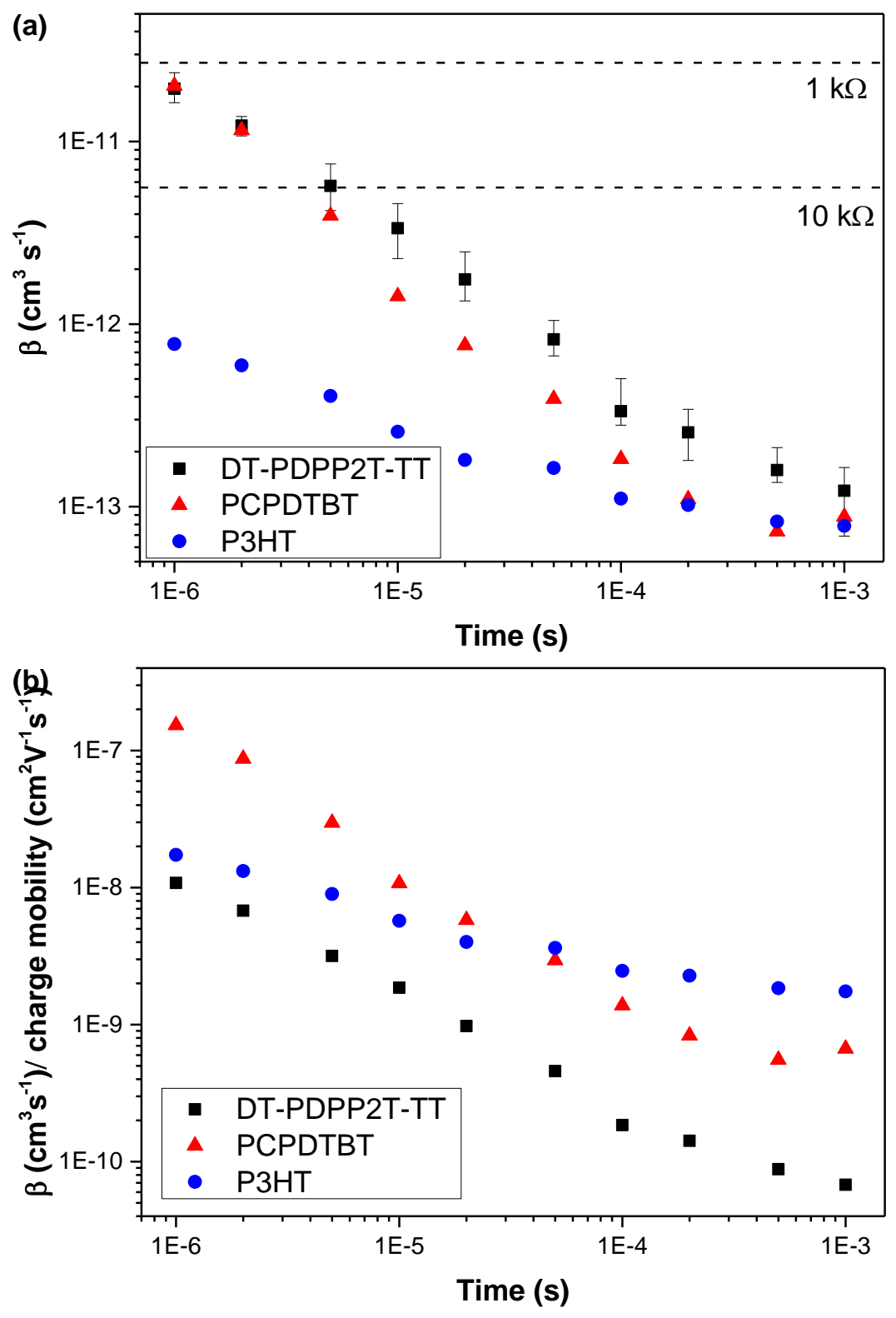

Figure 4. The time dependence of bimolecular recombination coefficient $\beta$ in DT-PDPP2TTT: PCBM, PCPDTBT: PCBM and P3HT: PCBM devices. (a) $\beta$ plotted as a function of time, where the dash lines indicate $\beta$ values obtained from TOF, and (b) $\beta$ normalized to charge mobility, plotted as a function of time.

To compare the effect of dielectric screening on $\beta$ independently, the ratio between $\beta$ and charge mobility $\mu$ is plotted as a function of time (Figure $4 \mathrm{~b}$ ). It is observed that the DT- 
PDPP2T-TT:PCBM device, which has the highest dielectric constant, has the lowest values of $\beta / \mu$ throughout the investigated timescale. The P3HT:PCBM device has a $\beta / \mu$ value twice as that of DT-DPP2T-TT:PCBM at $1 \mu \mathrm{s}$, and the difference between the two systems increases at longer times due to the weaker time dependence in P3HT:PCBM. The PCPDTBT:PCBM device has $\beta / \mu$ values approximately an order of magnitude larger than that of DT-PDPP2TTT:PCBM throughout the timescale of $1 \mu \mathrm{s}$ to $1 \mathrm{~ms}$.

\subsection{Charge carrier lifetime}

The $V_{O C}$ dependent charge carrier lifetime $\tau$ is compared between the polymer: PCBM blends of DT-PDPP2T-TT, PCPDTBT and P3HT in Figure 5. To check the lifetime dependence on active layer thickness, P3HT:PCBM devices with thickness in the range of $67 \mathrm{~nm}$ to $306 \mathrm{~nm}$ as well as DT-PDPP2T-TT:PCBM devices with $185 \mathrm{~nm}$ and $267 \mathrm{~nm}$ active layer are compared. At $V_{O C}$ above $0.55 \mathrm{~V}$, the carrier lifetime dependence on thickness is within $10 \%$ deviation for both P3HT:PCBM and DT-PDPP2T-TT:PCBM systems and the thickness-dependence gradually increase at lower $V_{O C}$. At the maximum $V_{O C}$ for each system, DT-PDPP2TTT:PCBM and P3HT:PCBM devices show similar $\tau$ around $3 \mu$ s, while for the PCPDTBT:PCBM device a shorter $\tau$ of $1.3 \mu$ s is observed. At $V_{O C}$ below $0.7 \mathrm{~V}$, DT-PDPP2TTT:PCBM device showed the longest carrier lifetime throughout the investigated $V_{O C}$ range. A logarithmic dependence of $\tau$ on $V_{O C}$ is observed for all three systems at $V_{O C}$ above $0.55 \mathrm{~V}$. P3HT: PCBM showed the weakest $V_{O C}$ dependence of $\tau$, while DT-PDPP2T-TT: PCBM showed the strongest. 


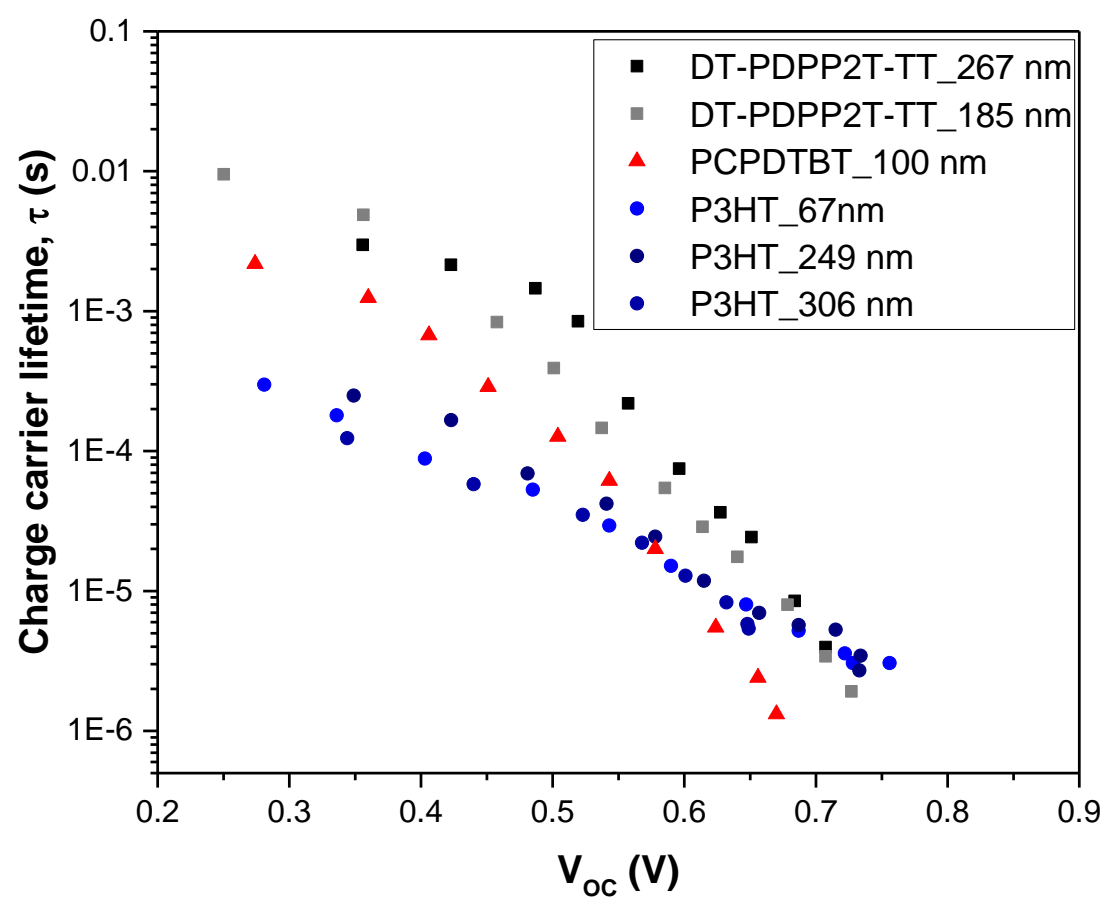

Figure 5. Charge carrier lifetime plotted as a function of open circuit voltage for DT-PDPP2TTT:PCBM, PCPDTBT:PCBM and P3HT:PCBM devices of different active layer thicknesses.

\section{Discussion}

\subsection{Increased dielectric screening in DT-PDPP2T-TT and DT-PDPP2T-TT:PCBM films}

The dielectric constant values measured by two different techniques for DT-PDPP2T-TT and DT-PDPP2T-TT:PCBM are the highest to our best knowledge in the literature. ${ }^{\left[1 \mathrm{c}, 1 \mathrm{e},{ }^{2-3,6]} \mathrm{We}\right.}$ have carefully checked that the results are not due to an experimental error or an oversight, by systematically checking the experimental conditions used in both electrical impedance and CELIV measurements. First the thickness dependence of the measured capacitance was checked to confirm that indeed the measured capacitance is the geometric capacitance of the sample described by Equation 6. Devices with five different active layer thicknesses in the range of 200 to $300 \mathrm{~nm}$ at the same blending ratio were fabricated, showing capacitance values 
inversely proportional to thickness. To check parasitic capacitance effect from neighbouring device pixels on the same substrates due to, for example, current flowing through the highly conductive PEDOT:PSS hole contact layer outside the patterned ITO electrode, devices with only one pixel were fabricated. These experiments yielded the same dielectric constant within $10 \%$ error. Applied bias-dependent capacitance measurements (Figure 1a) showed that while the pristine polymer devices displays a constant capacitance value at increasing reverse bias, the capacitance of a blend film devices decreases as the reverse bias is increased. No significant frequency dependence has been observed at applied biases between $-2 \mathrm{~V}$ and $2 \mathrm{~V}$ (Figure 1b); therefore no significant dispersion in the $R C$ time constant is observed. The more pronounced bias dependent capacitance in the donor: acceptor blend compared to the pristine polymer is hence attributed to double injection (electron and hole) and subsequent recombination, which decreases the measured capacitance. Small injection current $(10 \mu \mathrm{A})$ in the reverse bias was also observed in dark CELIV curves caused by the slight increase in the current response to a voltage ramp, while a purely capacitive current leads to a constant current. To minimise the effect of dielectric screening from reverse bias injection or leakage current, the capacitance in blend devices were taken at zero applied bias by extrapolation, a procedure frequently applied in the literature to obtained geometric capacitance. ${ }^{[14]}$

The CELIV transients for each device is recorded under a wide range of maximum voltage altitude $U$ between $0.2 \mathrm{~V}$ and $5 \mathrm{~V}$ and pulse duration $t_{\text {pulse }}$ between $10 \mu \mathrm{s}$ and $100 \mu \mathrm{s}$, allowing the bias voltage ramp ( $\left.d U / d t_{\text {pulse }}\right)$ to vary over an order of magnitude.

To summarise, a high dielectric constant of DT-PDPP2T-TT and DT-PDPP2T-TT:PCBM has been consistently obtained using two different techniques under every experimental conditions trialled. The dielectric constant value measured for the 1:3 w/w DT-PDPP2T-TT:PCBM blend is the approximate volume average of the dielectric constant value of the pristine polymer 
(16.7) and [60]PCBM (3.9), assuming same material density. We have measured the dielectric constant value for a 1:3 w/w DT-PDPP2T-TT:[70]PCBM blend, which was also found to be the volume average of the two materials (assuming that the dielectric constant of [70]PCBM is the same as [60]PCBM).

Volume-average dielectric constant in a bulk heterojunction blend has been reported before ${ }^{[15]}$ while in annealed P3HT:PCBM, the measured dielectric constant was higher than the calculated volume average dielectric constant at PCBM concentration below 40\%wt. ${ }^{[16]}$ This lack of agreement was explained by the non-uniform distribution of spherical PCBM domains in P3HT:PCBM layer at low PCBM concentration, as well as a PCBM-rich phase near the PEDOT:PSS surface. ${ }^{[17]}$ Furthermore, the deviation of dielectric constant from the calculated volume-average value has been correlated with the larger crystalline domain size of P3HT at low PCBM concentrations. Our observation of volume average dielectric constant suggests similar morphology of the polymer phase in the blend compared to the pristine film, diluted by lower dielectric constant PCBM molecules and crystallites. We preformed dielectric measurements on the same chemical structure polymer with lower molecular weight and found much smaller dielectric constant values than reported here. While we currently have no explanation of why this particular polymer and polymer:PCBM blend has high dielectric constant, the correlation with charge recombination and transport would be very interesting.

The above discussion raises some interesting aspects of dielectric screening in donor:acceptor bends with a large asymmetry between dielectric constant values. A volume-averaged dielectric constant measured by low frequency dielectric spectroscopic techniques is may not be sufficient to describe localized (on the nm scale) dielectric screening of photo-excited and charge separated states, particularly in highly crystalline films with 10-20 nm domain size as it was reported for DT-PDPP2T-TT:PCBM. ${ }^{[5 c]}$ The specific arrangement at the interface 
between the large dielectric constant polymer and fullerene, such as lamellar orientation, domain size and domain purity may all influence dielectric screening. As a specific example, dielectric screening in a DT-PDPP2T-TT-rich phase with high crystallinity will likely to be much stronger than in a PCBM-rich phase. Such special variation of dielectric screening strength should be more pronounced in DT-PDPP2T-TT:PCBM than in P3HT:PCBM, due to the four times larger difference between polymer and PCBM dielectric constant. Such an effect may help to confine charge carriers within ordered domains, screening from charge carrier traps located at the phase boundary, further affecting charge mobility and recombination dynamics. Clearly, further investigations are required in this area and DT-PDPP2T-TT:PCBM is a very good candidate for such studies.

\subsection{The effect of increased dielectric screening on recombination kinetics and device performance}

Figure $4 \mathrm{~b}$ shows that when normalized to charge carrier mobility, DT-PDPP2T-TT:PCBM system has the lowest bimolecular recombination to mobility ratio. This could be explained by two different mechanisms in the framework of diffusion-controlled bimolecular recombination models. These are the effect of dielectric screening on i) coulomb capture radius; and ii) its possible effect on the bimolecular reduction factor $\zeta$. Firstly, increased dielectric screening in DT-PDPP2T-TT:PCBM $\quad\left(\varepsilon_{r}=7.2\right.$ versus $\varepsilon_{r}=3.5$ for P3HT:PCBM and $\varepsilon_{r}=3.3$ for PCPDTBT:PCBM) leads to a smaller coulomb capture radius $r_{c}$ of $2 \mathrm{~nm}(4.4 \mathrm{~nm}$ for P3HT:PCBM, and $4.1 \mathrm{~nm}$ for PCPDTBT:PCBM (Table S1). In turn, the smaller radius leads to elongated beta as it takes longer for the electrons and holes to diffuse close to each other to be captured by their mutual Coulomb interaction. Note that faster charge transport leads to faster diffusion of the charges, which has been excluded in this analysis by normalizing the 
measured $\beta$ to mobility. The second mechanism is related to bimolecular recombination reduction factor $\zeta$, which itself may have multiple origins. Arkhipov et al. suggested that recombination at donor-acceptor interface is slowed by an energetic barrier, formed by the presence of interfacial dipoles in an ordered phase. ${ }^{[18]}$ Recombination via tail states has been suggested as another mechanism for reduced bimolecular recombination, in which case the recombination rate is suggested to be activation-controlled rather than diffusion. ${ }^{[9 a, 19]}$ Another mechanism by Hilczer and Tachiya suggests that recombination of electron and holes leads to an intermediate CT state, which again can separate to free charges. ${ }^{[20]}$ Therefore, electron and hole encounters do not always lead to charge annihilation. ${ }^{[21]}$ Explanations based largely on the morphology, such as 2D Langevin recombination ${ }^{[22]}$ has also been put forward suggesting reduced probability for charges to meet in highly ordered lamella or fibril-like structured P3HT:PCBM films.

To separate the two possible origins, first the bimolecular reduction factor $\zeta$ is calculated from bulk generation TOF measurements. For the DT-PDPP2T-TT:PCBM system, a reduction factor of 0.07 was obtained using a $1 \mathrm{k} \Omega$ resistor (Figure $\mathrm{S} 2$ ). This value is not as small as previously reported non-Langevin systems measured at similar conditions, for example $10^{-4}$ for P3HT:PCBM ${ }^{[13]}$ and 0.02 for a silole-based polymer PDTSiTTz:PCBM. ${ }^{[23]}$ Furthermore, the TOF transients decay closely following an exponential decay, without the appearance of a distinct extraction time $t_{e}$. The $Q_{e} / C U_{0}$ ratio is close to 1 , which has been observed in blends featuring Langevin-type recombination; ${ }^{[24]}$ whereas for donor/acceptor blends with reducedLangevin recombination, this ratio could be as large as $30 .{ }^{[25]}$ Photo-CELIV transients at saturated intensity also clearly suggest Langevin recombination, where the extracted charge equals the capacitive charge $\left(\frac{Q_{e}}{C U}=1.1\right)$ and $t_{\max }$ is related to transit time. Note that reduced 
bimolecular recombination leads to photo-CELIV transients with extracted charge significantly larger than the capacitive charge, which could be easily observed in a large $\Delta j / j(0)$ ratio above ten. $^{[26]}$ Also, in systems with non-Langevin bimolecular recombination, the maximum extraction time $t_{\max }$ will be controlled by the extraction time rather than the transit time, thus decreasing $t_{\max }$ at longer delay times will be expected. ${ }^{[23 \mathrm{~b}]}$ In summary, our results indicate a bimolecular recombination process that is very close to diffusion controlled, Langevin-type ${ }^{[25]}$ for the DT-PDPP2T-TT:PCBM. Consequently, the possible effect of stronger dielectric screening on the reduction factor will not be discussed further.

In the case of diffusion dominated recombination, the diffusion coefficient $D$ is determined only by the mobility of the charge carriers following Einstein's relation: ${ }^{[27]}$

$D=\mu k_{B} T / e$

This gives a diffusion coefficient of $4.6 \times 10^{-5} \mathrm{~cm}^{2} \mathrm{~s}^{-1}$ for DT-PDPP2T-TT: PCBM, and 3.4 $\times 10^{-}$ ${ }^{6}$ and $1.2 \times 10^{-6} \mathrm{~cm}^{2} \mathrm{~s}^{-1}$ for PCPDTBT: PCBM and P3HT: PCBM, respectively. Assuming even distribution of charge carriers within the active layer and using the obtained charge density values at $1 \mu \mathrm{s}\left(1 \times 10^{16}, 3.5 \times 10^{16}\right.$ and $3 \times 10^{17} \mathrm{~cm}^{-3}$ for DT-PDPP2T-T:PCBM, PCPDTBT:PCBM and P3HT:PCBM, respectively), the time it takes for charges to diffuse over a $5 \mathrm{~nm}$ distance is $290 \mathrm{~ns}, 1.5 \mu \mathrm{s}$ and $2.9 \mu$ s for DT-PDPP2T-TT:PCBM, PCPDTBT:PCBM and P3HT:PCBM, respectively. The calculated diffusion time in DT-PDPP2T-TT:PCBM device is a significantly shorter, agreeing with the high diffusion coefficient originated from high carrier mobility. We note that outside the column capture radius at open circuit voltage, charges are likely to follow some random path so the average diffusion distance for electrons and holes could be larger than the typical domain size. Nevertheless, an interesting observation is that only in DT-PDPP2TTT:PCBM system is the calculated diffusion time over $5 \mathrm{~nm}$ distance significantly shorter than the carrier lifetime of $4 \mu \mathrm{s}$. The carrier lifetime of $1.1 \mu \mathrm{s}$ in PCPDTBT:PCBM is shorter than 
the diffusion time and the charge lifetime of $3.1 \mu$ s in P3HT:PCBM is slightly longer, but comparable to the charge diffusion time. This is a clear indication that despite the fast charge diffusion in DT-PDPP2T-TT:PCBM devices, the probability for charges to meet and recombine is reduced. This is explained not by the reduction factor (see above), but by the smaller coulomb capture radius due to increased dielectric screening.

The smaller $\beta / \mu$ explained by increased dielectric screening is therefore particularly beneficial for systems with high charge carrier mobility and showing Langevin-type recombination, such as the DT-PDPP2T-TT:PCBM. Enhanced dielectric screening balances the increased $\beta$ caused by high mobility, as a result, DT-PDPP2T-TT:PCBM and PCPDTBT:PCBM show very similar $\beta$ at $1 \mu$ s despite DT-PDPP2T-TT:PCBM having a mobility one order of magnitude higher. Higher mobility leads to faster diffusion-controlled recombination rate, but the decreased coulomb capture radius partially compensate for the faster diffusion. .Note that the longer charge carrier lifetime observed in DT-PDPP2T-TT:PCBM (Figure 5) compared to PCPDTBT:PCBM blend is due to the smaller $\beta$. Compared to P3HT:PCBM blend, on the other hand, the longer lifetime is attributed to the lower extracted charge carrier density at the same open circuit voltage. This could be explained by the much larger density of trap states in P3HT:PCBM blends used in this study, also evident from the rather low charge mobility, the shallow slope of Voc versus charge density and the highly dispersive nature of charge recombination kinetics at long delay time (Figure S3). To summarize, under current extraction conditions, charges diffuse under the gradient of the electrochemical potential faster than the recombination lifetime, explaining high fill factor at large active layer thicknesses compared to both PCPDTBT:PCBM and P3HT:PCBM blends. 
A question arises whether the increased dielectric screening is responsible for the higher mobility in DT-PDPP2T-TT:PCBM. Mobility dependence on electric field in disordered mediate can be described by the Poole-Frenkel model

$\mu(T, E)=\mu_{0} \exp \left(-\frac{E_{0}-\beta_{P F} F^{\frac{1}{2}}}{k_{B} T_{e f f}}\right)$

where $\mu_{0}$ is the field-independent mobility, $E_{0}$ is the zero-field activation energy, $\beta_{P F}$ is the Poole-Frenkel coefficient and $T_{\text {eff }}^{-1}=T^{-1}-T_{0}^{-1}$ where $T$ is the temperature and $T_{0}$ is the extrapolation intercept of temperature dependent mobility plots. ${ }^{[28]}$ The $\mu_{0}$ describes the mobility in the absence of disorder in an ideal matrix with no distribution of site energies. The electric field dependence arises from the variation of site energies and the effect of external field on the activation barrier for charge carrier hops. ${ }^{[29]}$

If the dielectric constant increase is due to larger polarizability of the electronical conductivity by the delocalized segments of the conjugated chains, for example, due to larger bandwidth of interchain coupling, increased mobility due to larger, extended delocalization is expected, (increased $\mu_{0}$ ). Furthermore, increased dielectric screening may affect the average electrochemical energy of the polarons in the conductive polymer, which in turn may affect the activation energy for charge carrier hops. In both cases, the increase in dielectric screening and charge mobility are inherently linked. Increasing the dielectric constant is therefore partially counterproductive in systems with Langevin-type diffusion controlled bimolecular recombination, as the smaller Coulomb radius is partially compensated by the faster diffusion. Still, there are net befits as demonstrated in this manuscript.

If the larger dialectic constant is due to the movement of oriented sidechain dipoles (as it is suspected in this case), delocalization within the electronically active conductive segments is not necessarily effected, therefore $\mu_{0}$ is not changed. Variation of site energies, often termed 
energetic disorder, may change instead. It was suggested that increased dielectric screening effectively smooths the energy landscape in a disordered system, leading to faster transport. ${ }^{[30]}$ We note these arguments are based on a tridimensional uniform dielectric screening, which is unlikely to be the case. Charges primarily conducted within the polymer phase experience larger dielectric screening than charges at the interface, which may confine them within the phase and away from the interface. This could lead to faster transport as well as slower recombination. Studies of the effect of dielectric screening on charge transport and recombination in strongly reduced, non-Langevin recombination systems will be very interesting.

\section{Conclusion}

A dielectric constant of seven and a charge mobility of $1.8 \times 10^{-3} \mathrm{~cm}^{2} \mathrm{~V}^{-1} \mathrm{~s}^{-1}$ is measured in a DTPDPP2T-TT:[60]PCBM polymer solar cell devices, both of these values are among the highest reported in the literature to date. The bimolecular recombination kinetics is studied in this blend and results are compared to PCPDTBT:PCBM and P3HT:PCBM blends. Bimolecular recombination coefficient close to PCPDTBT is observed at early timescales using charge extraction with nanosecond switch techniques, despite the order of magnitude higher charge mobility. The reduction factor $\beta / \beta_{L}$ of the observed recombination kinetics compared to recombination predicted by Langevin theory was found to be similar to that of P3HT:PCBM. The results reported here suggest that the increased dielectric screening reduces charge recombination explained by the smaller coulomb capture radius. The improved dielectric screening balances the faster diffusion arising from higher charge mobility, leading increased charge extraction efficiencies at large active layer thicknesses. The studies here raises some important questions, such as the origin of the improved dielectric constant in DT-PDPP2T-TT 
and its fullerene blends as well as the effect of dielectric screening ion bimolecular recombination in non-Langevin type recombination systems.

\section{Experimental Section}

Device Fabrication and Current-Voltage Characterization: DT-PDPP2T-TT was purchased from 1-Material (OS0300, Lot\# YY6278). PCPDTBT, P3HT and [60]PCBM were purchased from Solaris. Photovoltaic devices were fabricated as follows: pre-patterned indium tin oxide (ITO) substrates with active area of $0.06 \mathrm{~cm}^{2}$ were cleaned by sonicating in detergent, DI water, acetone and isopropanol, each for $15 \mathrm{~min}$ then blow dry with nitrogen and treated with UVOzone for twenty minutes. Poly-(ethylenedioxythiophene): poly(styrene sulfonate) (PEDOT:PSS) (Clevios P, VP Al 4083) was spin coated on the substrates then subsequently annealed at $140^{\circ} \mathrm{C}$ for ten minutes to achieve a dry thickness of around $30 \mathrm{~nm}$. The photoactive layer was deposited via spin coating the hot solution (DT-PDPP2T-TT:[60]PCBM with 1:3 w/w ratio and pristine DT-PDPP2T-TT in chloroform with 7.5 vol\% dichlorobenzene; PCPDTBT:[60]PCBM with $1: 2 \mathrm{w} / \mathrm{w}$ ratio in chlorobenzene with 3 vol\% diodooctane; P3HT:[60]PCBM with 1:1 w/w ratio in chlorobenzene). Active layer thickness was controlled via changing spin speed and measured using Dektak stylus profilometer. The above processes are carried out under ambient condition except polymer solution preparation, which was prepared in an Argon filled glovebox. Thermal annealing was carried out for P3HT device at $110^{\circ} \mathrm{C}$ for ten minutes in glovebox. Aluminum counter electrode was deposited by thermal evaporation at $\sim 1 \times 10^{-6}$ mbar to achieve thickness around $100 \mathrm{~nm}$. The complete devices were encapsulated with cover glass using UV-curable epoxy in glovebox for characterizations under ambient conditions. Current-Voltage characterizations were carried out using Solar cell $I-V$ curve testing system model IV21L, PV measurements.

Film thickness: Film thickness was measured using DEKTAK stylus profilometer. Each measurement was carried out over a scan length of $3000 \mu \mathrm{m}$ with a stylus force of $0.1 \mathrm{mg}$. For each sample multiple scans were carried out for an averaged thickness.

Bias and frequency dependent capacitance: Voltage and frequency dependent capacitance was measured using a Zahner IM6 electrochemical workstation and a Solartron 1287 electrochemical interface. AC oscillation amplitude of $5 \mathrm{mV}$ was used. The voltage bias between $-2 \mathrm{~V}$ and $2 \mathrm{~V}$ and frequency between $1 \mathrm{kHz}$ and $500 \mathrm{kHz}$ was applied. 
Charge Extraction by Linearly Increasing Voltage: CELIV was carried out using a function generator (WF 1974, NF) and the extraction current is recorded using an oscilloscope over 50 $\Omega$ impedance.

Photogenerated Charge Extraction by Linearly Increasing Voltage: A pulsed laser (532nm, Spectra Physics Quanta Ray Lab 170) is used to generate charge carriers within measured devices. A Quanta Ray MOPO was used to tune the pump wavelength for photoexcitation. Excitation pulse of $650 \mathrm{~nm}$ was used for DT-PDPP2T-TT:PCBM and PCPDTBT:PCBM devices and $532 \mathrm{~nm}$ for P3HT:PCBM devices. The delay time between photoexcitation and voltage ramp application is controlled using a nanosecond switch (2 M $\Omega$ impedance, Asama Lab) and a delay generator (Stanford Research DG535). The extraction current is recorded using an oscilloscope, using either the build-in $50 \Omega$ impedance on oscilloscope or a homemade $13.5 \Omega$ resistor in parallel with the $1 \mathrm{M} \Omega$ input impedance on oscilloscope, depending on the device capacitance.

Bulk Generation Time-of-Flight: The device was photoexcited using a laser pulse same as above while biased by applying $2 \mathrm{~V}$ reverse bias using a function generator. The extraction currents were recorded using an oscilloscope. The circuit impedance is controlled by applying resistors in parallel to the $1 \mathrm{M} \Omega$ input impedance of oscilloscope. The excitation density of the device is altered by using different neutral density filters, with the highest excitation density of $1000 \mu \mathrm{Jcm}^{-2}$.

Time-Resolved Charge Extraction: TRCE measurements were carried out as follows. A pulsed laser (532 nm, Spectra Physics Quanta Ray Lab 170) was used to generate charge carriers within measured devises. A Quanta Ray MOPO was used to tune the pump wavelength for photoexcitation. Excitation pulse of $650 \mathrm{~nm}$ was used for DT-PDPP2T-TT devices and $532 \mathrm{~nm}$ for P3HT devices. The devices were initially held at high impedance using a nanosecond switch 
(2 M $\Omega$ impedance, Asama Lab). After a controllable delay time using a digital delay generator

(Stanford Research DG535), the switch is opened and the devices are at short circuit condition.

The extracted current is measured using an oscilloscope as a function of time (input impedance $50 \Omega)$. The dark response is subtracted to remove the switch noise.

Photovoltage decay: The devices were photoexcited at open circuit (1 $\mathrm{M} \Omega$ impedance). The voltage decay was recorded as a function of time. The extracted charge is then matched with $V_{O C}$ at each delay time.

\section{Supporting Information}

Supporting Information is available from the Wiley Online Library or from the author.

\section{Acknowledgements}

The authors thank Shogo Mori for helpful discussions. This research was supported under the Australian Research Council's Linkage Projects funding scheme. A.J.M. acknowledges the ARC for providing equipment support through LIEF, as well as supporting A.J.M. and T.M.C. with an Australian Research Fellowship and DECRA fellowship, respectively.

Received: ((will be filled in by the editorial staff))

Revised: ((will be filled in by the editorial staff))

Published online: ((will be filled in by the editorial staff))

[1] a) S. A. Hawks, F. Deledalle, J. Yao, D. G. Rebois, G. Li, J. Nelson, Y. Yang, T. Kirchartz, J. R. Durrant, Adv. Energy Mater. 2013, 3, 1201; b) L. J. A. Koster, V. D. Mihailetchi, P. W. M. Blom, Appl. Phys. Lett. 2006, 88, 093511; c) P. Y. Yang, M. J. Yuan, D. F. Zeigler, S. E. Watkins, J. A. Lee, C. K. Luscombe, J Mater Chem C 2014, 2, 3278; d) L. J. A. Koster, S. E. Shaheen, J. C. Hummelen, Adv. Energy Mater. 2012, 2, 1246; e) Y. Lu, Z. Xiao, Y. Yuan, H. Wu, Z. An, Y. Hou, C. Gao, J. Huang, J. Mater. Chem. C 2013, 1,630 .

[2] S. Torabi, F. Jahani, I. Van Severen, C. Kanimozhi, S. Patil, R. W. a. Havenith, R. C. Chiechi, L. Lutsen, D. J. M. Vanderzande, T. J. Cleij, J. C. Hummelen, L. J. A. Koster, Adv. Funct. Mater. 2015, 25, 150. 
[3] S. Chen, S. W. Tsang, T. H. Lai, J. R. Reynolds, F. So, Adv Mater 2014, 26, 6125.

[4] a) J. H. Hou, H. Y. Chen, S. Q. Zhang, G. Li, Y. Yang, J. Am. Chem. Soc. 2008, 130, 16144; b) H. Y. Chen, J. H. Hou, S. Q. Zhang, Y. Y. Liang, G. W. Yang, Y. Yang, L. P. Yu, Y. Wu, G. Li, Nat. Photonics 2009, 3, 649; c) Y. Y. Liang, Z. Xu, J. B. Xia, S. T. Tsai, Y. Wu, G. Li, C. Ray, L. P. Yu, Adv. Mater. 2010, 22, 135; d) S. H. Park, A. Roy, S. Beaupre, S. Cho, N. Coates, J. S. Moon, D. Moses, M. Leclerc, K. Lee, A. J. Heeger, Nat. Photonics 2009, 3, 297.

[5] a) M. S. Kim, B. G. Kim, J. Kim, ACS Appl. Mater. Inter. 2009, 1, 1264; b) J. Peet, L. Wen, P. Byrne, S. Rodman, K. Forberich, Y. Shao, N. Drolet, R. Gaudiana, G. Dennler, D. Waller, Appl. Phys. Lett. 2011, 98; c) W. Li, K. H. Hendriks, W. S. C. Roelofs, Y. Kim, M. M. Wienk, R. a. J. Janssen, Adv. Mater. 2013, 25, 3182; d) S. C. Price, A. C. Stuart, L. Q. Yang, H. X. Zhou, W. You, J. Am. Chem. Soc. 2011, 133, 4625; e) W. W. Li, A. Furlan, K. H. Hendriks, M. M. Wienk, R. A. J. Janssen, J. Am. Chem. Soc. 2013, 135, 5529; f) X. G. Guo, N. J. Zhou, S. J. Lou, J. Smith, D. B. Tice, J. W. Hennek, R. P. Ortiz, J. T. L. Navarrete, S. Y. Li, J. Strzalka, L. X. Chen, R. P. H. Chang, A. Facchetti, T. J. Marks, Nat. Photonics 2013, 7, 825; g) Y. Liu, J. Zhao, Z. Li, C. Mu, W. Ma, H. Hu, K. Jiang, H. Lin, H. Ade, H. Yan, Nat Commun 2014, 5, 5293; h) Z. Chen, P. Cai, J. Chen, X. Liu, L. Zhang, L. Lan, J. Peng, Y. Ma, Y. Cao, Adv. Mater. 2014, 26, 2586; i) M. Zhang, X. Guo, W. Ma, H. Ade, J. Hou, Adv. Mater. 2014, 26, 5880; j) T. L. Nguyen, H. Choi, S. J. Ko, M. A. Uddin, B. Walker, S. Yum, J. E. Jeong, M. H. Yun, T. J. Shin, S. Hwang, J. Y. Kim, H. Y. Woo, Energy Environ. Sci. 2014, 7, 3040; k) V. Vohra, K. Kawashima, T. Kakara, T. Koganezawa, I. Osaka, K. Takimiya, H. Murata, Nat. Photonics 2015, 9, 403.

[6] N. Cho, C. W. Schlenker, K. M. Knesting, P. Koelsch, H. L. Yip, D. S. Ginger, A. K. Y. Jen, Adv. Energy Mater. 2014, 4, 1301857.

[7] M. Guo, T. Hayakawa, M. A. Kakimoto, T. Goodson III, J Phys. Chem. B 2011, 115, 13419.

[8] W. W. Li, K. H. Hendriks, W. S. C. Roelofs, Y. Kim, M. M. Wienk, R. A. J. Janssen, Adv. Mater. 2013, 25, 3182.

[9] a) A. J. Ferguson, N. Kopidakis, S. E. Shaheen, G. Rumbles, J Phys. Chem. C 2011, 115, 23134; b) F. C. Jamieson, T. Agostinelli, H. Azimi, J. Nelson, J. R. Durrant, $J$ Phys. Chem. Lett. 2010, 1, 3306.

[10] J. A. Carr, S. Chaudhary, Appl. Phys. Lett. 2012, 100, 2.

[11] A. J. Mozer, G. Dennler, N. S. Sariciftci, M. Westerling, A. Pivrikas, R. Österbacka, G. Juška, Phys. Rev. B 2005, 72, 035217.

[12] T. M. Clarke, C. Lungenschmied, J. Peet, N. Drolet, A. J. Mozer, Adv. Energy Mater. 2015, 5, 1401345.

[13] A. Pivrikas, G. Juška, A. J. Mozer, M. Scharber, K. Arlauskas, N. Sariciftci, H. Stubb, R. Österbacka, Phys. Rev. Lett. 2005, 94, 176806.

[14] a) S. Nowy, W. Ren, A. Elschner, W. Lovenich, W. Brutting, J. Appl. Phys. 2010, 107, 054501; b) M. Mingebach, C. Deibel, V. Dyakonov, Phys. Rev. B 2011, 84, 153201.

[15] a) B. Bernardo, D. Cheyns, B. Verreet, R. D. Schaller, B. P. Rand, N. C. Giebink, Nat Commun. 2014, 5, 3245; b) P. Yang, M. Yuan, D. F. Zeigler, S. E. Watkins, J. A. Lee, C. K. Luscombe, J. Mater. Chem. C 2014, 2, 3278.

[16] S. Engmann, V. Turkovic, G. Gobsch, H. Hoppe, Adv. Energy Mater. 2011, 1, 684. 
[17] a) P. G. Karagiannidis, D. Georgiou, C. Pitsalidis, A. Laskarakis, S. Logothetidis, Mater. Chem. Phys. 2011, 129, 1207; b) S. Engmann, C. R. Singh, V. Turkovic, H. Hoppe, G. Gobsch, Adv. Energy Mater. 2013, 3, 1463.

[18] V. I. Arkhipov, E. V. Emelianova, H. Bassler, Phys. Rev. Lett. 1999, 82, 1321.

[19] a) G. Garcia-Belmonte, J. Bisquert, Appl. Phys. Lett. 2010, 96, 113301; b) T. Kirchartz, B. E. Pieters, J. Kirkpatrick, U. Rau, J. Nelson, Phys. Rev. B 2011, 83, 115209.

[20] a) M. Hilczer, M. Tachiya, J. Phys. Chem. C 2010, 114, 6808; b) T. M. Burke, S. Sweetnam, K. Vandewal, M. D. McGehee, Adv. Energy Mater. 2015, 5,1500123.

[21] S. Yamamoto, H. Ohkita, H. Benten, S. Ito, J. Phys. Chem. C 2012, 116, 14804.

[22] a) M. Nyman, O. J. Sandberg, R. Österbacka, Adv. Energy Mater. 2015, 5, 1400890; b) R. Österbacka, A. Pivrikas, G. Juška, A. Poškus, H. Aarnio, G. Sliaužys, K. Genevičius, K. Arlauskas, N. S. Sariciftci, IEEE J. Sel. Top. Quantum Electron. 2010, 16, 1738.

[23] a) T. M. Clarke, J. Peet, P. Denk, G. Dennler, C. Lungenschmied, A. J. Mozer, Energy Environ. Sci. 2012, 5, 5241; b) T. M. Clarke, D. B. Rodovsky, A. A. Herzing, J. Peet, G. Dennler, D. DeLongchamp, C. Lungenschmied, A. J. Mozer, Adv. Energy Mater. 2011, $1,1062$.

[24] T. M. Clarke, J. Peet, A. Nattestad, N. Drolet, G. Dennler, C. Lungenschmied, M. Leclerc, A. J. Mozer, Org. Electron. 2012, 13, 2639.

[25] A. Pivrikas, N. S. Sariciftci, G. Juska, R. Osterbacka, Prog. Photovoltaics 2007, 15, 677.

[26] A. Armin, M. Velusamy, P. L. Burn, P. Meredith, A. Pivrikas, Appl. Phys. Lett. 2012, 101.

[27] G. Lakhwani, A. Rao, R. H. Friend, Annual review of physical chemistry 2014, 65, 557.

[28] W. D. Gill, J. Appl. Phys 1972, 43, 5033.

[29] H. Bässler, Phys. Status Solidi (b) 1993, 175, 15.

[30] a) A. E. Jailaubekov, A. P. Willard, J. R. Tritsch, W.-L. Chan, N. Sai, R. Gearba, L. G. Kaake, K. J. Williams, K. Leung, P. J. Rossky, X. Y. Zhu, Nat. Mater. 2013, 12, 66; b) C. Wan, Y. Kodama, M. Kondo, R. Sasai, X. Qian, X. Gu, K. Koga, K. Yabuki, R. Yang, K. Koumoto, Nano Lett 2015, 15, 6302. 
A high dielectric constant of seven and charge mobility of $1.8 \times 10^{-3} \mathrm{~cm}^{2} \mathrm{~V}^{-1} \mathrm{~s}^{-1}$ is reported for a low bandgap polymer DT-PDPP2T-TT:PCBM blend. Reduced bimolecular recombination normalized to mobility is observed, which is explained by smaller coulomb capture radius in a diffusion controlled recombination mechanism.

Keyword: dielectric screening, polymers, organic solar cell, recombination

Guanran Zhang, Tracey M. Clarke* and Attila J. Mozer*

Bimolecular recombination in a low bandgap polymer: PCBM blend solar cell with a high dielectric constant

ToC figure

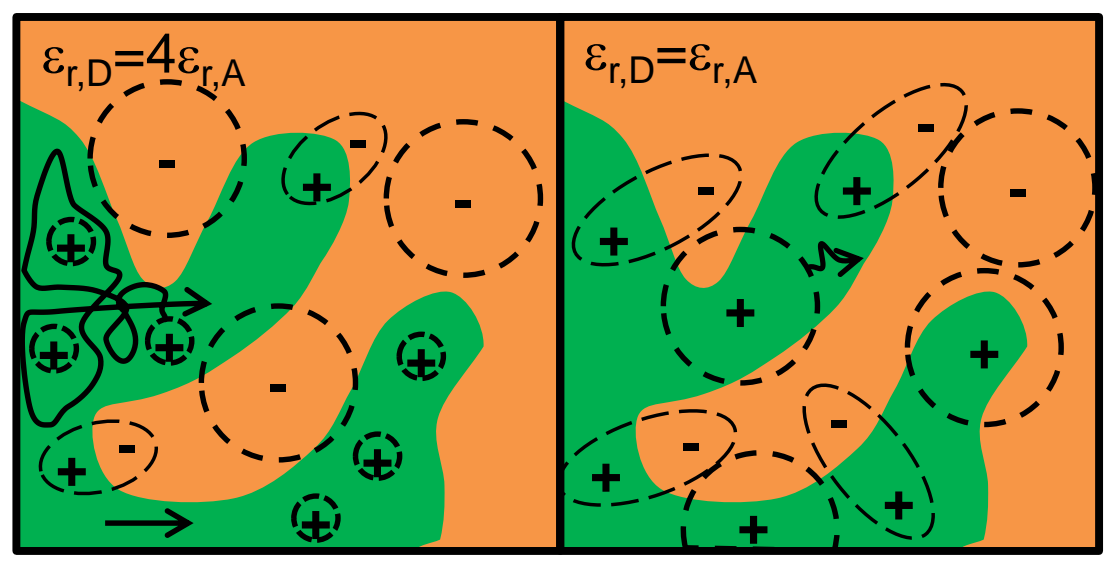


Copyright WILEY-VCH Verlag GmbH \& Co. KGaA, 69469 Weinheim, Germany, 2013.

Supporting Information

Bimolecular recombination in a low bandgap polymer: PCBM blend solar cell with a high dielectric constant

Guanran Zhang, Tracey M. Clarke* and Attila J. Mozer*

Table S1. Coulomb capture radius and charge dissociation probability calculated at different dielectric constant and film thicknesses

\begin{tabular}{lccc}
\hline Dielectric constant, $\varepsilon_{r}$ & $\begin{array}{l}\text { Film thickness } \\
(\mathrm{nm})\end{array}$ & $\begin{array}{l}\text { Coulomb capture radius, } r_{c} \\
(\mathrm{~nm})\end{array}$ & Dissociation probability $P(E)$ \\
\hline 3 & 100 & 4.8 & $1.1 \times 10^{-4}$ \\
& 300 & & $8.2 \times 10^{-5}$ \\
7 & 100 & 2.1 & $2.1 \times 10^{-2}$ \\
& 300 & & $1.8 \times 10^{-2}$ \\
10 & 100 & 1.4 & $6.7 \times 10^{-2}$ \\
& 300 & & $6.0 \times 10^{-2}$ \\
\hline
\end{tabular}



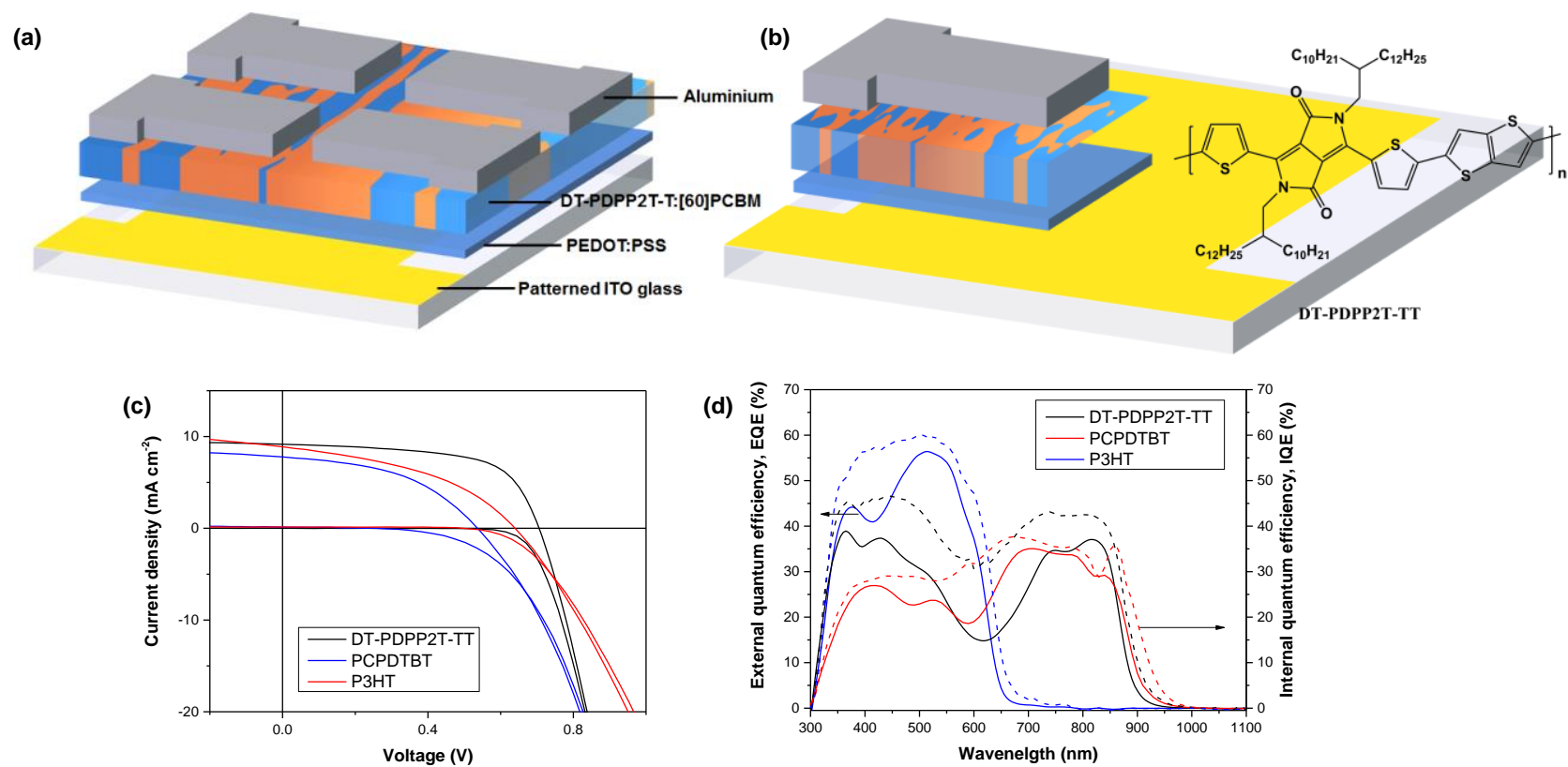

Figure S1. The chemical structure of DT-PDPP2T-TT and (a) device architecture used for DTPDPP2T-TT: PCBM devices, (b) single-pixel device architecture used for dielectric constant measurements, (c) current density-voltage characterizations of DT-PDPP2T-TT:PCBM, PCPDTBT:PCBM and P3HT:PCBM devices, and (d) internal/external quantum efficiency characterizations for the three devices measured in (c).

Table S2. A list of device parameters for dielectric constant calculation.

\begin{tabular}{lccc}
\hline Acceptor & $\begin{array}{c}\text { Thickness } \\
(\mathrm{nm})\end{array}$ & $\begin{array}{c}\text { Capacitance } \\
(\mathrm{nF})\end{array}$ & $\begin{array}{c}\text { Dielectric } \\
\text { constant }\end{array}$ \\
\hline [60]PCBM & $200 \pm 12$ & $1.99 \pm 0.09$ & $7.47 \pm 0.33$ \\
[60]PCBM & $250 \pm 13$ & $1.52 \pm 0.30$ & $7.15 \pm 0.40$ \\
[60]PCBM & $267 \pm 8$ & $1.35 \pm 0.05$ & $6.79 \pm 0.14$ \\
[60]PCBM & $295 \pm 16$ & $1.45 \pm 0.08^{\mathrm{a})}$ & $8.04 \pm 0.43^{\mathrm{a})}$ \\
[60]PCBM & $300 \pm 11$ & $1.24 \pm 0.14^{\mathrm{a})}$ & $7.02 \pm 0.75^{\mathrm{a})}$ \\
[70]PCBM & $107 \pm 15$ & $3.40 \pm 0.40$ & $6.85 \pm 0.88$
\end{tabular}

\footnotetext{
a) Values measured from single pixel devices, where the counter electrode is only evaporated on a single pixel area (see Figure S1b)
} 
Table S3. Photovoltaic performance parameters of DT-PDPP2T-TT:PCBM, PCPDTBT:PCBM and P3HT:PCBM devices.

\begin{tabular}{lcccccc}
\hline Polymer & $\begin{array}{c}\mathrm{D}: \mathrm{A} \\
\text { ratio }\end{array}$ & $\begin{array}{c}\mathrm{V}_{\mathrm{OC}} \\
(\mathrm{V})\end{array}$ & $\begin{array}{c}\mathrm{J}_{\mathrm{SC}} \\
\left(\mathrm{mA} \mathrm{cm}^{-2}\right)\end{array}$ & $\begin{array}{c}\mathrm{J}_{\mathrm{EQE}} \\
\left(\mathrm{mA} \mathrm{cm}^{-2}\right)\end{array}$ & $\begin{array}{c}\text { Fill } \\
\text { Factor }\end{array}$ & Efficiency \\
\hline DT-PDPP2T-TT & $1: 3$ & 0.71 & 9.16 & 9.1 & 0.62 & $4.0 \%$ \\
PCPDTBT & $1: 2$ & 0.64 & 8.89 & 9.0 & 0.42 & $2.4 \%$ \\
P3HT & $1: 1$ & 0.54 & 7.78 & 7.2 & 0.46 & $1.9 \%$ \\
\hline
\end{tabular}
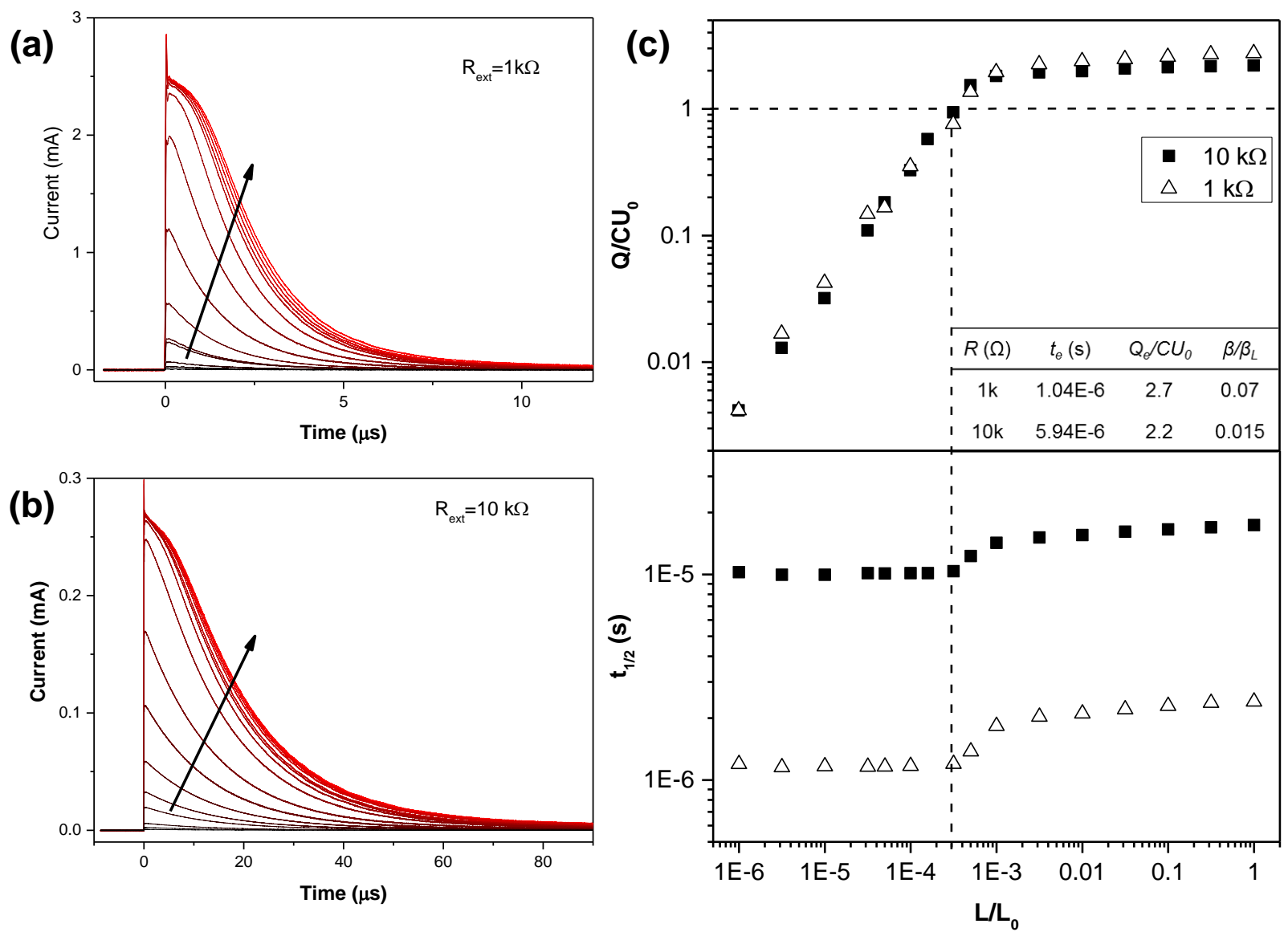

Figure S2. Bulk-generation time-of-flight results measured under external resistance of $1 \mathrm{k} \Omega$ and $10 \mathrm{k} \Omega$. The obtained TOF transients under different excitation densities (increasing excitation density indicated by the direction of the arrow) are shown for (a) $1 \mathrm{k} \Omega$ resistance and (b) $10 \mathrm{k} \Omega$ resistance, and (c) the extracted $Q_{e} / C U_{0}$ and $t_{1 / 2}$ are plotted as function of excitation density. 


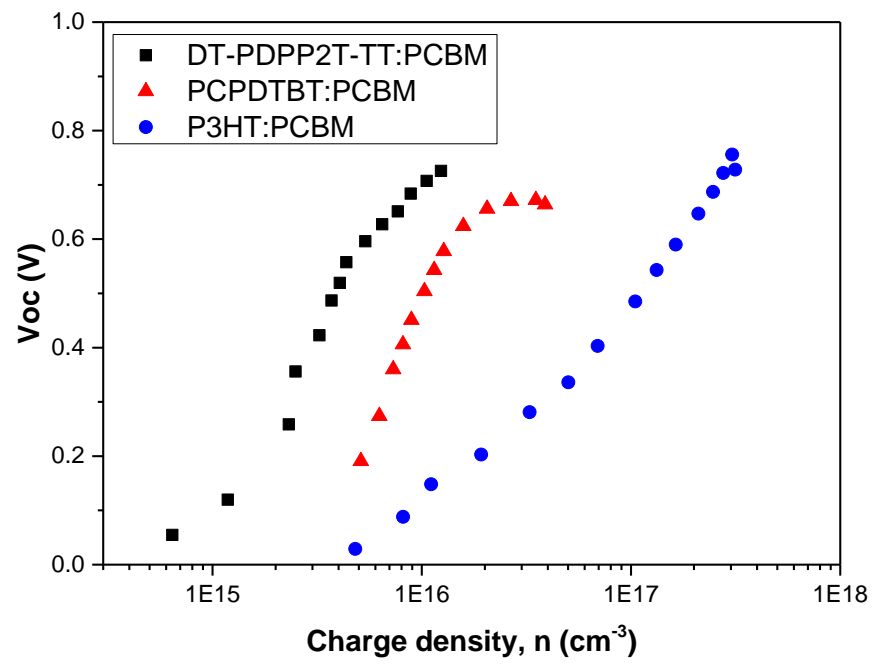

Figure S3. The open circuit voltage behavior with charge density for DT-PDPP2T-TT:PCMB, PCPDTBT:PCBM and P3HT:PCBM devices, shown at saturated excitation density. 